\title{
Charles Dobzynski, du réalisme à l'imaginaire
}

\author{
Monique W. Labidoire
}

$\mathrm{L}$

es sources profondes de l'œuvre de Charles Dobzynski jaillissent de lieux multiples, tous d'importance, dans lesquels voisinent langue, humanisme, spiritualité, conscience. Sa langue qui est d'abord d'essence poétique est aussi langue de communication, deux langages qu'il crée pour comprendre et donner, pour transmettre et révéler.

S'il y a errance chez Charles Dobzynski c'est peut-être dans la recherche d'une ou de plusieurs formes poétiques qu'il conquiert dans une liberté pas toujours visible immédiatement mais qui se révèle dans le creusement approfondi de l'écriture. En introduction à son livre La vie est un orchestre, il nous donne un aperçu d'un art poétique qui pourrait nous laisser peu d'espoir sur l'avenir de la poésie :

\section{J'écris}

J'écris. Je creuse un trou. La glace est dure

Que forme le langage en son lac.

Un jour on m'enfermera dans un sac

Pour me jeter avec d'autres ordures.

Mais il poursuit et nous rassure :

On couvrira mon corps avec des lettres Je rêve d'un lexique pour linceul. Un alphabet ne meurt pas pour un seul Mot qui tira son avoir de son être.

\section{(La vie est un orchestre, 7 )}

Être poète, c'est l'assurance du seul bien possédé et produit par soimême, du seul bien à offrir à autrui dans l'espace-temps accordé à chacun 
d'entre nous. Charles Dobzynski n'est pas particulièrement à la recherche de la postérité. Et il sait que les ordures sont du monde. Personne n'y échappera. La vie est un orchestre: nous dit-il avec ce titre qu'il y a dans la vie une fosse peuplée d'exécutants dirigés par un chef ou au contraire un ensemble qui exécute dans l'harmonie et la paix une partition de vie qu'on ne peut jouer seul. Il y a sans doute plusieurs interprétations possibles et le poète dispose de notre curiosité avec une certaine jubilation. Mais c'est vers sa propre curiosité que nous conduit notre lecture. C'est vers cette étonnante énergie que le poète manifeste par l'écoute et par le regard et qu'il nous propose d'adopter, que nous avançons avec lui, comme si son désir était seulement du poème, de l'homme et de la lumière. Des voies qui nous intéressent.

Du chant d'Orphée aux claquettes du rêve américain en passant par Jerry Mulligan et les Rolling stones, le poème roule sa vie rythmée en écho à une réalité qui ne devrait pas nous échapper. Malgré l'ordure, la vie est belle et mérite d'être chantée.

Le monde est un chant peuplé de mots et Charles Dobzynski ne tourne pas autour du pot. Il emploie formes et mots qui conviennent à son poème, des mots comme formica, hémiplégie, tuyauterie ou encore des initiales adoptées par tous au quotidien comme R.E.R ou B.D. que l'on ne s'attend pas à trouver dans une poésie. Mais justement, nous sommes dans le poème et non pas dans les poésies. Nous sommes dans la particularité d'une écriture qui n'évite pas la langue vernaculaire contemporaine, qu'elle soit des faubourgs ou de l'université.

Ici, les images d'un Bunuel investissent le poème pour le soutenir dans un combat contre l'hypocrisie, ainsi du charme discret de la poésie à celui d'une société à laquelle le poète n'adhère sans doute pas, nous retrouvons un surréalisme qui nous traverse de part en part dans un des multiples lieux du poète où l'imaginaire et la réalité sont confrontés à des possibilités nouvelles qui n'ont pas encore d'existence propre mais qui sont pressenties par le poète.

Là, Charles Dobzynski revient au noyau dur de sa poésie, le noyau de la conscience et du combat dans lequel seule la tolérance pourra guérir les plaies toujours ouvertes. Les étranges étrangers, plus étranges encore s'ils sont poètes, pourraient, devraient, être les premiers à s'installer à « la table d'écoute " pour tendre l'oreille à tous ceux qui prennent le train de la destinée :

Signes d'adieu. Regards héliotropes.

Gens de partout qui s'en vont vers l'ailleurs, 


\section{L'air de l'Atlas alcoolise l'Europe}

Le train transmue à ses roues d'orpailleur

Toute distance et toute destinée. [...]

(La vie est un orchestre, 27)

Il y a des sonorités rimbaldiennes chez Charles Dobzynski, une façon d'épanouir son poème en bousculant quelques convenances - poétiques dont nous avons tant besoin dans ce microcosme de l'écriture qui nous donne beaucoup, beaucoup trop de poétiquement correct. :

\section{La vie-orchestre}

Il est des jours sans mesure. On y muse

Et l'été tinte aux doigts mezzo-voce

On prend le vert d'un roman de Ramuz.

Le ciel se tait. Le futur est fauché.

Et puis sifflante la musique afflue

Dans les cafés cliquetis de flippers,

Le juke-box magnétise le flux

Les sons font des signes d'auto-stoppeurs

Quelle alchimie a distillé leur drogue?

(La vie est un orchestre, 16)

Il n'y a pas un côté noir où tout serait ténèbres et un côté blanc où tout serait lumière. Il y a des circuits de vie où se métissent les couleurs, les langues, les amours. Les passages se font dans le poème et par le poème, dans l'engagement et l'action, dans la réflexion et la conscience. Le poète passe l'épreuve du feu et de la foi, liant à l'intérieur de l'éprouvette de son vécu tous ses trésors récoltés depuis l'enfance et qu'il a acquis grâce à sa naissance au cœur de l'Europe, son exil, son identité, sa différence, trésors de persévérance, de révolte, de culture native et de culture acquise, de rites et de traditions, trésors toujours, qui lui permettent d'avancer.

De la Vistule à l'Ourcq, des poèmes sortis d'une mémoire matricielle tous attachés au cordon de la vie par un immense amour et un grand respect à la mère. Mère, nourricière d'amour, qui conduit le fils vers l'amour des autres et guide le poème vers l'ouverture et vers l'amour des autres femmes. On sait l'importance d'une mère dans l'image que le futur homme aura des femmes.

Tu es ma seeur. Et je t'aime. Ma fermme

Celle qui m'a chaque jour accouché 
Ma mère un peu. Peut-être aussi la flamme

Qui dans ton corps si jose la toucher

Brûle mes mots ces pauvres noctuelles

Mes souvenirs que plus rien ne défend

Miroir en quoi l'image est virtuelle

Je te regarde et tu es mon enfant.

\title{
(La vie est un orchestre, 67)
}

Des buanderies de Charles Dobzynksi au lavoir d'Attilà Joszef il n'y a que deux langues différentes mais le même langage poétique, la mémoire reste intacte, vivante dans le présent du poème de l'un comme de l'autre. Elle nous rapproche de notre propre mémoire et nous aide à éclairer les parties plus obscures de nos souvenirs personnels.

Il y a sûrement eu dans l'existence de Charles Dobzynski communication essentielle avec les mots, ce qui nous ramène à la "Table d'écoute " que le poète donne en sous-titre à son livre important qu'est Table des Éléments. Écouter les mots, écouter les éléments c'est comprendre une musique intérieure, c'est creuser et fouiller l'univers à l'aide du seul outil dont le poète puisse se servir, le poème. Avec "Sonate des mots ", nous sommes dans l'élégiaque, dans le lyrisme, dans la passion. Le poète est ivre de la vie des mots et nous dégustons à petites gorgées cette ivresse qui nous conduit à la joie, tout de même bien rare, d'être enfin dans un élément que nous reconnaissons comme bon pour nous. La langue créée par le frisson et le sensible nous porte vers quelque chose qui a à voir avec le sensuel, la jouissance et le bonheur. Le corps des mots reçoit le don de vie :

\section{Avec les mots on fait l'amour Ils tombent \\ De nos étreintes [...]}

\section{(Table des Éléments, 7)}

Des étreintes parfois douloureuses c'est vrai, ce serait trop simple s'il n'y avait que joie. Il y a une telle profusion et une telle richesse dans la poésie de Charles Dobzynski que l'on est comme devant un coffre rempli à ras bords de mille objets, de mille sujets qui nous enveloppent.

Autant de mots

\author{
Autant de vide \\ Autant de vie \\ Autant de trous [...] \\ (Table des Eléments, 15)
}


Le temps, l'atome, la terre. Des sonates. Des thèmes à deux voix, du poète et du temps, du poète et de l'atome, du poète et de la terre, qui peuvent être jouées par un petit ensemble qui comprendrait aussi le lecteur, une voix off, une pièce plus intérieure au poème si c'était encore possible.

\section{Vide comment \\ parler au vide surpeuplé? [...]}

(Table des Éléments, 30)

Et pourtant ces sonates s'ouvrent vers un estuaire pour gagner les grands espaces de la mer et de l'océan, les horizons de l'espace et de l'univers. Mais aussi ceux de la mer océane matrice de la vie et de la mort et de tous les recommencements possibles :

\section{[...] De ma naissance à ma mort tempes de mer sans paupière l'oeil de l'écume ouvre ma vie aux scintillements aveugles de l'océan.}

\section{(Table des Éléments, 118)}

Agir, créer, c'est ordonner un chaos, c'est remplir la page du poème de signes qui construiront le lieu de vie et le lieu de mort. C'est rassembler le temps dans une surface nourrie d'inventions nécessaires pour une naissance chaque fois recommencée. Le poète va jusqu’à glorifier cette nécessité comme dans sa "Sonate des céréales » où la symbolique du le pain rejoint toutes les eucharisties, où l'éternité ne semble trouver réponse que par la renaissance des éléments et des richesses de la nature qui sont offertes à l'homme. Seigle, blé, épeautre, orge, tout un langage premier de vie germinale dans le partage du poème et du pain, nourritures essentielles au poète :

\section{La nuit}

\section{Les céréales}

occupent tout l'obscur

de la planète [...]

Maille à maille

nous filons

l'écheveau de maïs 
Fil de naissance

Fil de mort

en nos mains le métissage

La paille du futur crépite

du soleil

or de l'oubli

mil de l'absence

un seul filon de durée

l'épi se mue en pépite. [...]

(Table des Éléments, 120)

Ce n'est pas parce que le corps du monde ne peut contenir tout l'or de ses éléments que le poète va se décourager. Il peut tenter une autre aventure, plus intime et encore plus proche de sa propre réalité. La quête identitaire plus universelle va passer au particulier, un particulier qui l'a toujours habité. Nous reprenons avec le poète le chemin qu'il a suivi de la Vistule à l'Ourcq et nous pouvons nous arrêter un moment avec lui. Nous sommes entre Belleville et La Villette, nous descendons vers République et la rue des Rosiers. Le pain au pavot est gonflé de mémoire yiddish et les cornichons doux de saumure laissent dans l'encre du poète quelques traces fécondes. Dans les échoppes, des odeurs, des étoffes, des sons, tissent un peuple. Un grondement, des blessures et le poète restera dans "Les ombres du vécu " :

\author{
Dans un wagon plombé \\ Je roule \\ avec des ombres, \\ jour et nuit dans l'oubli \\ le mimétisme, \\ pas de balise \\ sur l'océan des cendres [...]
}

(Alphabase, 15)

Charles Dobzynski nous parle d'un " destin sans destinataire " et l'identification, il dit encore plus puisqu'il emploie le mot mimétisme, est un état fréquent que l'on a relevé chez les très proches des victimes de toutes les infamies. Des camps nazis aux geôles de Pinochet à d'autres lieux encore trop nombreux de la barbarie, des questions se sont élevées sur le pourquoi, le comment et le soi-même, questions qui resteront toujours sans réponses.

Le poème, s'il ne trouve pas réponse définitive peut ouvrir une brèche dans les barbelés et confisquer une certaine culpabilité. Dans Alphabase, Charles Dobzynski pratique une liturgie peut-être un peu thérapeutique en 
nommant les plaies. Un baume adoucissant va être étendu sur les blessures mais les cicatrices resteront visibles. Chaque lettre de l'alphabet hébreu sera signe de mémoire et de survie. Parlant de cet alphabet le poète écrit dans son introduction "Certaines de ces lettres, comme parfois les étoiles ont leur double, un signe qui n'est utilisé qu'en finale pour clore un mot. c'est l'ultime wagon que l'on accroche au train pour un voyage sans retour".

Mais le destin n'a-t-il pas toujours des destinataires? Comme le poème. Le voyage n'est pas sans retour dans la poésie de Charles Dobzynski. Il revient dans sa mémoire et dans nos mémoires. Le poète va prendre à bras le corps son destin en prenant un autre corps du monde, poétique cette fois, qu'il va ensemencer de mots, de rythmes, de sens. Il doit prendre quelque distance avec le réel et l'identité pour introduire dans son imaginaire des graines de survie. Il va danser dans son "Opéra de l'espace » et tourner mille fois sur lui-même à la manière du derviche-tourneur pour atteindre le possible. La matière est un chant et la poésie " une aventure colossable ${ }^{1}$.

Et puisque c'est une aventure elle nous raconte l'histoire d'explorateurs, l'histoire de cet explorateur qu'est le poète Charles Dobzynski. On ne s'y trompe pas. Le poème prend son envol dans une astronef, il médite sur le mutant, il devient le locataire de l'ordinateur et pas de trou noir. La lumière jaillit de toutes ces histoires qui nous émerveillent à la manière d'un conte de fées. Car il faut de la magie au poète.

La magie de la langue qui ne quitte jamais Charles Dobzynski.

Je suis ma langue
Je l'essuie

Sur moi sueur ronge

par tous mes pores

elle me secrète m'essaie

massaille [...]

Je prolifere

\author{
syllabes de sable 2 \\ quel désert les désassemble? [...] \\ (Délogiques, 13)
}

1. Guillevic.

2. Il s'agit bien d'un vers de Charles Dobzynski et non du titre du livre de Lionel Ray. 
A lire et relire ce poète nous sommes dans une certitude qui ne nous étreint pas si souvent, celle de l'espérance. A piocher dans la matière poétique, à soigner la pierre comme le moindre éclat de soleil, à abreuver terre et syllabes de sables, nous avons encore la joie d'une révélation : celle de l'utilité du poème et du poète.

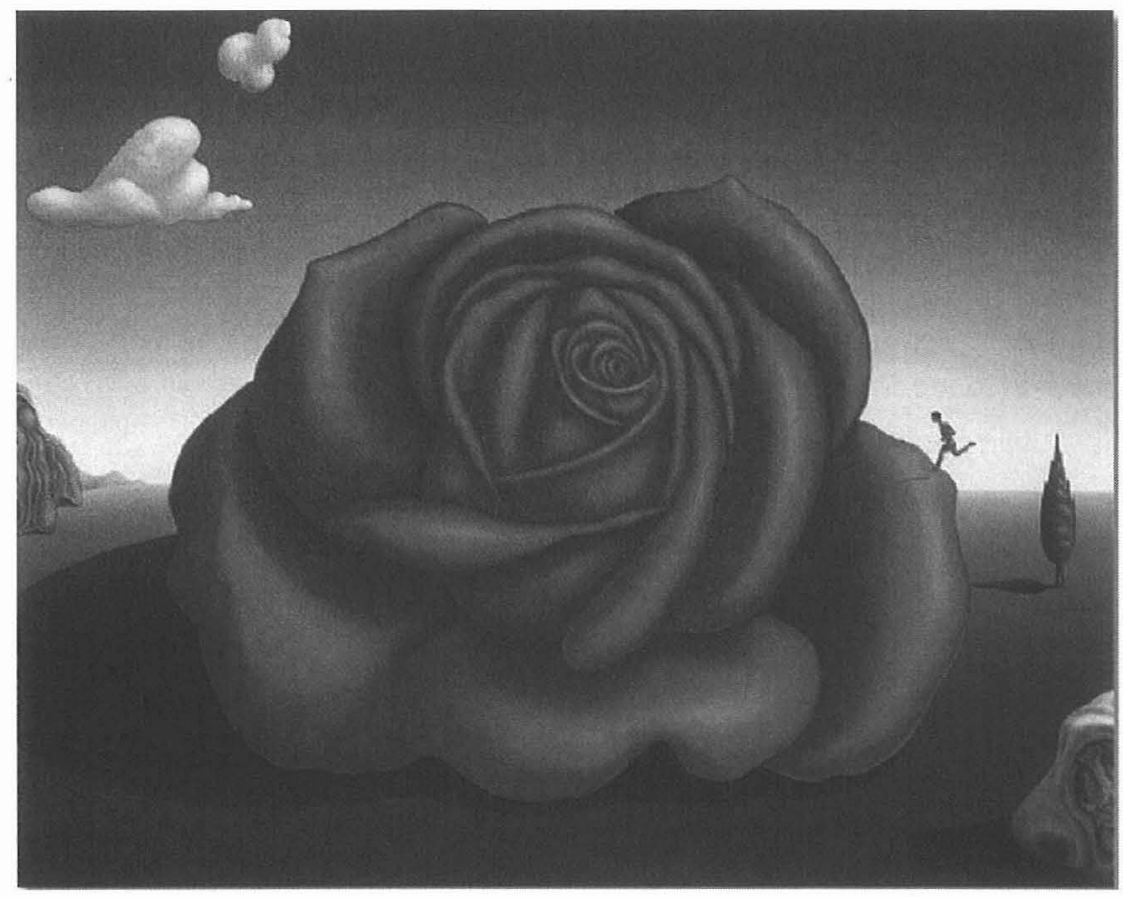

Joseph Cusimano, Eros'repose

huile sur toile, $1991,75 \mathrm{~cm}$ x $60 \mathrm{~cm}$ 